\title{
Energy Spectrum Measured by the Telescope Array Experiment
}

\author{
Dmitri Ivanov*, for the Telescope Array Collaboration ${ }^{\dagger}$ \\ University of Utah, Department of Physics \& Astronomy and High Energy Astrophysics Institute, \\ Salt Lake City, Utah, USA \\ E-mail: dmiivanov@gmail.com
}

\begin{abstract}
The Telescope Array (TA) is a cosmic ray detector in the Northern hemisphere that is sensitive to cosmic rays of energies ranging from $2 \mathrm{PeV}$ to $100 \mathrm{EeV}$ and higher. The main TA consists of two types of detectors: a ground array of 507 plastic scintillation counters, that covers an area of $700 \mathrm{~km}^{2}$ area on the ground, and a fluorescence detector (FD), distributed among 3 stations, that overlooks the ground array. The TA Low Energy Extension (TALE) consists of 10 additional fluorescence telescopes, at one of the TA FD stations, that are pointed to view higher elevation angles, and an infill array of 103 plastic scintillation counters in the field of view of the telescopes. The main TA measures cosmic rays of energies from $1 \mathrm{EeV}$ and higher, and the TALE extends the sensitivity of TA to $2 \mathrm{PeV}$. In this work, we summarize the latest TA and TALE spectrum measurements.
\end{abstract}

36th International Cosmic Ray Conference -ICRC2019-

July 24th - August 1st, 2019

Madison, WI, U.S.A.

\footnotetext{
* Speaker.

${ }^{\dagger}$ for collaboration list see PoS(ICRC2019)1177
} 


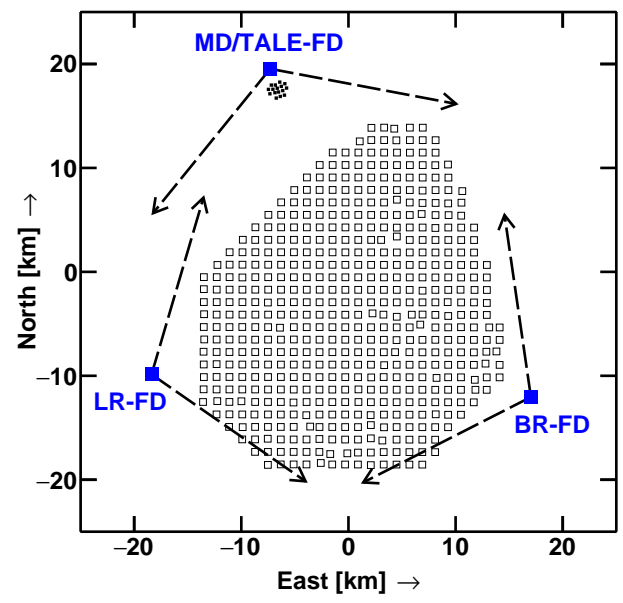

Figure 1: The Telescope Array detector. Open square boxes represent the locations of the main TA SD counters, small filled squares correspond to the TALE infill array counters, and large fill boxes show the TA fluorescence detector sites Black Rock Mesa (BR), Long Ridge (LR), and Middle Drum (MD), with their azimuthal viewing ranges represented by arrows.

\section{Introduction}

Telescope Array (TA) is a large hybrid cosmic ray detector that was deployed in 2007 in Millard County, UT, USA to observe ultrahigh energy cosmic rays in the Northern hemisphere. The main part of the experiment consists of a surface detector (SD) array that is overlooked by three fluorescence detector (FD) stations. TA SD [1] consists of 507 counters that are positioned on a square grid with $1200 \mathrm{~m}$ spacing and covering a total area of $\sim 700 \mathrm{~km}^{2}$ area on the ground. Each individual SD unit consists of 2 layers of $3 \mathrm{~m}^{2} \times 1.2 \mathrm{~cm}$ plastic scintillator.

The three TA FD stations are Black Rock Mesa (BR), Long Ridge (LR) [2], and Middle Drum (MD) [3]. TA BR and LR have 10 fluorescence telescopes, of 256 pixels each, that are viewing 3 to $33^{\circ}$ elevation range and covering a total azimuthal range of $2 \times 108^{\circ}$. The TA BR and LR fluorescence telescopes are equipped with $10 \mathrm{MHz}$ FADC readout electronics. The main TA MD site has 14256 pixel fluorescence telescopes, viewing 3 to $31^{\circ}$ in elevation and covering a total azimuthal range of $112^{\circ}$. TA Middle Drum uses sample and hold readout system.

The TA low energy extension (TALE) is also a hybrid detector, which consist of 10 additional HiRes-II [4] fluorescence telescopes added at the TA Middle Drum site to cover elevations from $31^{\circ}$ to $57^{\circ}$ and that overlook an infill array of 103 plastic scintillation counters. Each TALE telescope uses 256 pixels and a $10 \mathrm{MHz}$ FADC readout system. The TALE infill array has been fully deployed last year, while the TALE FD has been in full operation since 2013. Figure 1 summarizes the present configuration of the Telescope Array experiment. 


\section{TA Surface Detector Energy Spectrum}

\subsection{SD Spectrum Calculated Using Standard TA Procedure}

Figure 2 shows the TA surface detector spectrum, calculated using data collected over the time period from $2008 / 05 / 11$ to $2019 / 05 / 11$. Superimposed as a solid line is a fit to a broken power law function. We find the position of the ankle feature at $E_{A}=10^{18.69 \pm 0.01} \mathrm{eV}$, with powers before and after the ankle $p_{1}=-3.28 \pm 0.02$ and $p_{2}=-2.68 \pm 0.02$, respectively, the second break point, also known as the GZK cutoff [5, 6], at $E_{2}=10^{19.81 \pm 0.03} \mathrm{eV}$, and the power after the second break $p_{3}=-4.84 \pm 0.5$ We estimate the significance of the suppression to be $8.4 \sigma$ and the energy at which the measured integral flux becomes half of that in the absence of the cutoff [7] is $E_{1 / 2}=10^{19.79 \pm 0.04} \mathrm{eV}$.

The analysis used for calculating the result in Figure 2, described in $[8,9]$, consists of a time fit to determine the geometry of the cosmic ray shower, a lateral distribution fit to the AGASA lateral distribution function [10] to find the shower signal size $800 \mathrm{~m}$ from the shower axis $(S 800)$, an initial energy estimate from a CORSIKA QGSJET II.3 [11, 12, 13] surface detector Monte Carlo $[14,9]$, and calibration of the TA SD energy scale to the TA FD. Calibration of the SD energy scale to the TA FD is done by using an energy-independent calibration factor of $1 / 1.27$, as described in [16]. Recently, in [16], it has been demonstrated that the TA SD reconstruction approach yields a result that is within 3\% of the result obtained by applying the Constant Intensity Cut method [17] to the TA data at the highest energies.

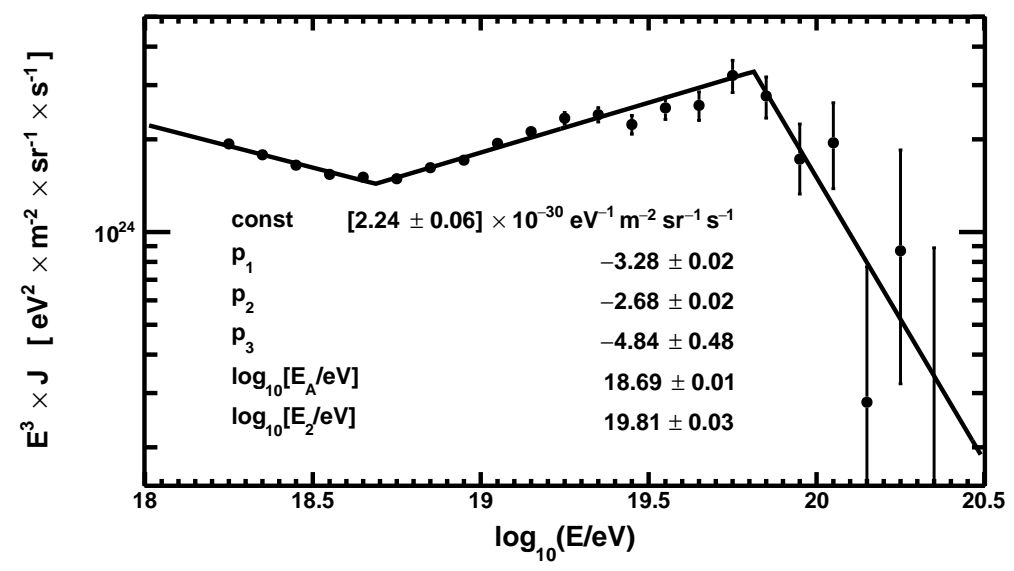

Figure 2: Telescope Array surface detector spectrum derived from 11 years of TA data, 2008/05/112019/05/11, using reconstruction described in [8,9]. Solid line is a fit to the broken power law, where $p_{1}, p_{2}$ are the spectral indices before and after the ankle, respectively, $E_{A}$ is the energy of the ankle, $E_{2}$ is the energy of the cutoff (aka the second break point), and $p_{3}$ is the spectral index after the cutoff. The significance of the cutoff at $10^{19.81} \mathrm{eV}$ has been estimated to be $8.4 \sigma$, and the energy at which the measured integral flux is half of that expected in the absence of the suppression, is $E_{1 / 2}=10^{19.79 \pm 0.04} \mathrm{eV}$.

\subsection{SD Spectrum Calculated Using Extended Quality Cuts}

The minimum energy threshold of the standard TA SD spectrum result, shown in Figure 2, 
is $10^{18.2} \mathrm{eV}$, where the efficiency of the TA SD is $\sim 10 \%$ of its value at the plateau. The event selection cuts, described in $[8,9]$, limit event zenith angles to a range from 0 to $45^{\circ}$. Above $10^{18.8}$ $\mathrm{eV}$, however, the TA SD is near the $100 \%$ efficiency point, allowing one to estimate the exposure by Monte Carlo accurately up to $55^{\circ}$ in zenith angle. Therefore, we can calculate the energy spectrum above $10^{18.8} \mathrm{eV}$ with higher statistical power by allowing the event zenith angles to be in 0 to $55^{\circ}$ range. After expanding the zenith angle range, and optimizing other quality cuts, first introduced in $[8,9]$, we arrive at the following list of cuts appropriate for the TA SD spectrum calculation above $10^{18.8} \mathrm{eV}$ (i) Event zenith angle is less than $55^{\circ}$, (ii) Each event includes at least 5 working counters that were hit (iii) Counter with largest signal is surrounded by 4 working counters on the square grid (iv) The uncertainty of the event pointing direction is less than $5^{\circ}(\mathrm{v})$ The fractional uncertainty of $S 800$ is less than $25 \%$ (vi) The reduced $\chi^{2}$ values of the time and lateral distribution are less than 4 . The TA SD spectrum with the above event selection cuts, over 11 years of data (2008/05/11-2019/05/11), is shown in the left panel of the Figure 3. As Figure 3 demonstrates, the spectrum with the new sets of quality cuts is consistent with the TA SD spectrum calculated using standard quality cuts $[8,9]$ at the $\sim 1 \%$ level.
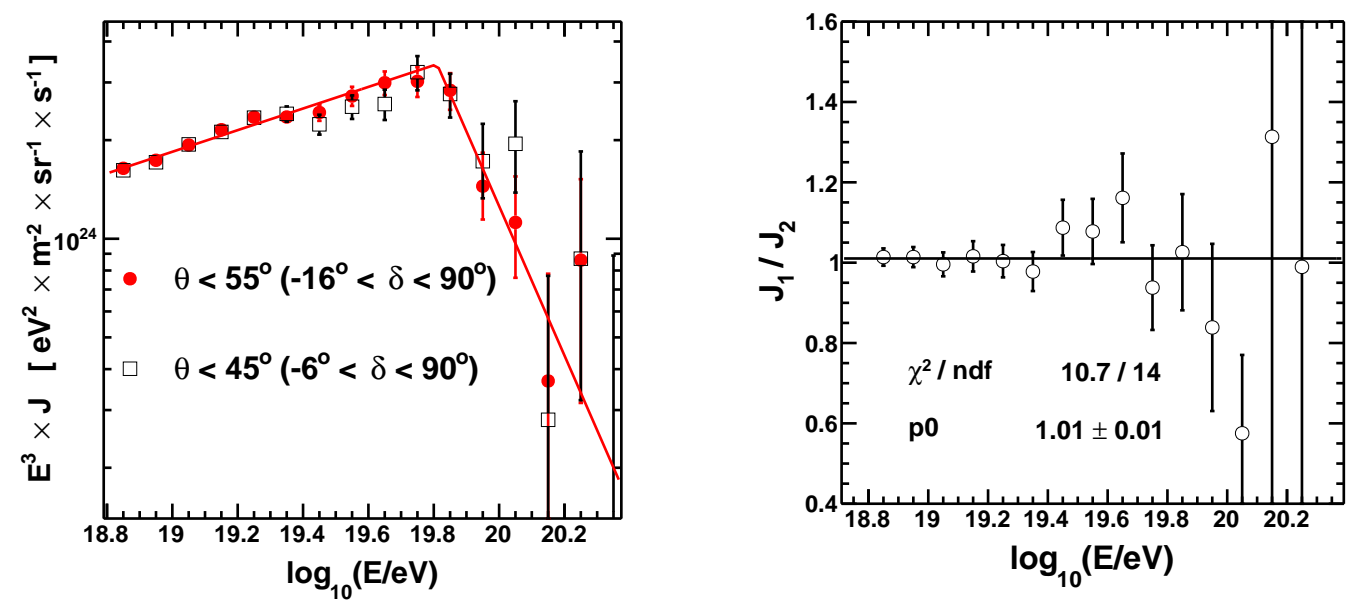

Figure 3: Left: Telescope Array surface detector energy spectra derived from 11 years of TA data, 2008/05/11-2019/05/11, using two sets of quality cuts. Red points show the result obtained using a new set of cuts described in the main text $\left(E^{3} J_{1}\right)$, which include zenith angle $\theta<55^{\circ}$ cut and cover a range of declinations $-16^{\circ}<\delta<90^{\circ}$. Black open squares show the result $\left(E^{3} J_{2}\right)$ obtained using the quality cuts described in [8,9], which include zenith angles up to $45^{\circ}$ and cover a range of declinations $-6^{\circ}<\delta<90^{\circ}$. Red line shows the fit to a broken power law function of the spectrum that uses zenith angles up to $55^{\circ}$. The fit finds the second break point at $10^{19.81 \pm 0.03} \mathrm{eV}$, and the spectral indices before and after the break point are $-2.67 \pm 0.02$ and $-5.3 \pm 0.5$, respectively. The significance of the cutoff at $10^{19.81 \pm 0.03} \mathrm{eV}$ has been estimated to be $12.0 \sigma$, and the energy at which the measured integral flux is half of that in the absence of the cutoff is $E_{1 / 2}=10^{19.77 \pm 0.02} \mathrm{eV}$. Right: A fit of the ratio of the two fluxes $J_{1}$ and $J_{2}$ to a horizontal line shows that the two results agree at a $\sim 1 \%$ level.

\subsection{Declination Dependence of the TA SD Spectrum}

Following the finding of the Auger-TA energy spectrum working group at the UHECR2016 conference that the cutoff energies of the TA and Auger spectra, initially disagreeing, come to a 
$0.6 \sigma$ agreement [15] when the measurements are restricted to the Auger-TA common declination band $\left(-15.7^{\circ}<\delta<24.8^{\circ}\right)$, we have found, in the first 7 years of TA SD data $(2008 / 05 / 11$ 2015/05/11), a $4 \sigma$ difference of the second break points [16] in the TA SD spectra calculated for the lower $\left(\delta<24.8^{\circ}\right)$ and higher $\left(\delta>24.8^{\circ}\right)$ declination bands of the TA SD field of view. Repeating this analysis for the SD data collected over the past 4 years, 2015/05/12 - 2019/05/11, we find that the difference between the two second break points of the spectra persists. As the left panel of Figure 4 shows, the second break point of the lower declination band spectrum is smaller than that of the higher declination band. The second break points are $10^{19.71 \pm 0.08} \mathrm{eV}$ and $10^{19.9 \pm 0.06} \mathrm{eV}$, for the lower and higher declinations, respectively, and are consistent with what has been reported for the first 7 years of the TA SD data in [16].
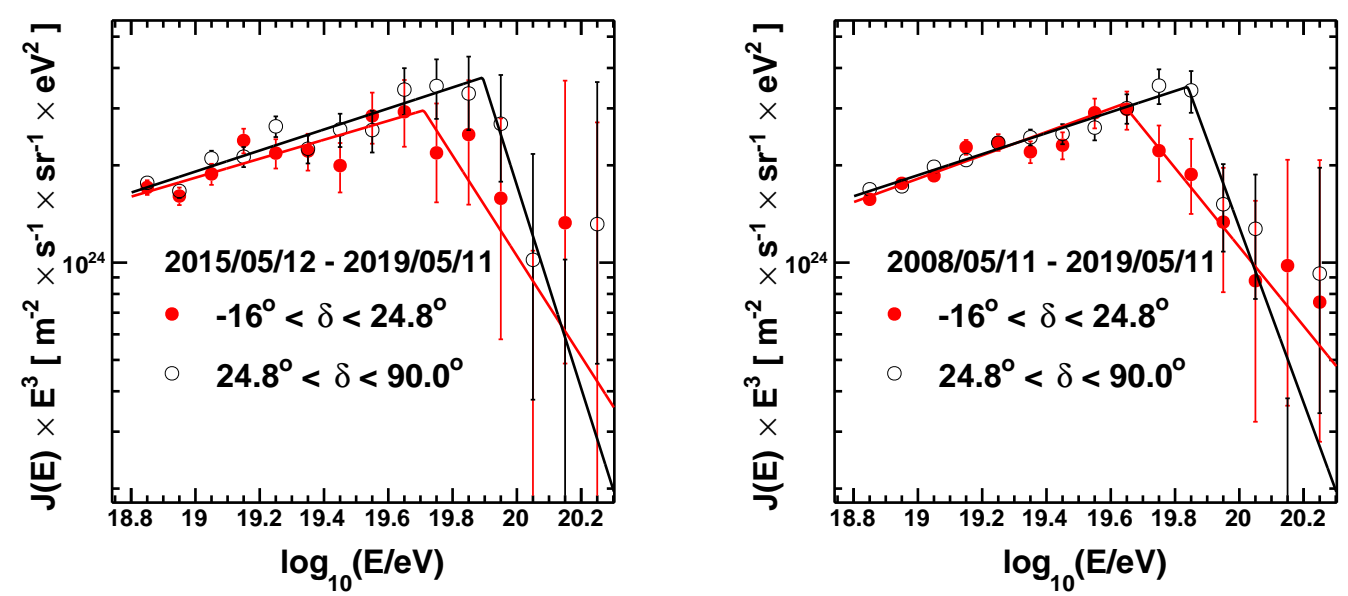

Figure 4: Left: TA SD energy spectrum collected in the last 4 years for the upper and lower declination bands. Solid lines show the fits to a broken power law function. The second break points for the lower and higher declination bands are $10^{19.71 \pm 0.08} \mathrm{eV}$ and $10^{19.9 \pm 0.06} \mathrm{eV}$, respectively. Right: TA SD energy spectrum collected over 11 years for the upper and lower declination bands. Superimposed are the fits to the broken power law functions. For the lower declination band, the spectral indices are $-2.64 \pm 0.04$ and $-4.2 \pm 0.3$ before and after the break, respectively, while the break point is at $10^{19.64 \pm 0.04} \mathrm{eV}$. For the higher declination band, the spectral indices before and after the break are $-2.67 \pm 0.03$ and $-5.71 \pm 0.6$, respectively, and the break point is at $10^{19.84 \pm 0.02} \mathrm{eV}$.

The combined TA SD data, over 11 years, yields the second break points of $10^{19.64 \pm 0.04} \mathrm{eV}$ for the lower declination band, and $10^{19.84 \pm 0.02} \mathrm{eV}$ for the higher declination band (see right panel of Figure 4. The local significance of this difference is $4.7 \sigma$. To estimate the global significance of this declination dependence effect, we have generated $10^{7}$ TA SD Monte Carlo samples, each of the size of the 11 years TA SD data (10356 events above $10^{18.8} \mathrm{eV}$ ), with one spectral cutoff energy, and counted trials where (i) the cutoffs in the declination bands $-16.0^{\circ}<\delta<24.8^{\circ}$ and $24.8^{\circ}<\delta<90^{\circ}$ are as significant (or more) as those in the data (48506 trials remaining), (ii) the break point energies of the two declination bands differ by more than $4.7 \sigma$ (438 trials remaining), (iii) the second break point in the lower declination band is within $1 \sigma$ from the Auger second break point in the TA/Auger common declination band [18] (98 trials remaining), and (iv) the second break point of the higher declination band spectrum $24.8^{\circ}<\delta<90^{\circ}$ is higher than the 
weighted average of the second break points of the full sky TA SD and HiRes spectra [4] (85 trials remaining). The total of 85 Monte Carlo trials, out of $10^{7}$, satisfy conditions (i-iv), which yields the global chance probability of the effect of $8.5 \times 10^{-6}$, or $4.3 \sigma$.

We have carried out extensive checks of systematic uncertainties of this result, for the first 7 years of data, which include checking for the detector biases by looking in the direction of the equatorial east and west instead of North and South in declination [18], comparison of the fluorescence detector and surface detector energies $[18,16]$, changing the reconstruction of the SD data to Constant Intensity Cut method [16], as well as examining the effects of using alternative hadronic interaction models in the reconstruction of the TA SD event energies [16]. In the process, we have constrained a possible energy nonlinearity bias of TA SD to $-0.3 \% \pm 9 \%$.

It is important to note that while the reported difference between the TA and Pierre Auger spectra in the common declination band amounts to an equivalent of a $20 \%$ per decade relative energy nonlinearity between the two experiments [19], even if this bias correction was applied entirely to the TA SD spectrum, it would not explain the difference of the second break point energies of the TA SD spectra measured in the lower and higher declination bands. To make the TA spectra agree between the lower and higher declination bands, different energy nonlinearity corrections would have to be applied to different event zenith angle ranges. In doing so, however, one would violate the agreement of the equatorial east and west of the TA SD spectrum comparison, first described in [18], and updated to the 11 years SD data in Figure 5. The equatorial east and west energy spectra, and their cutoff energies must be identical. This is a stringent test of the systematic biases.

\section{Combined TA Spectrum}

Figure 6 shows the combined TA spectrum using black filled circles. Energies below $10^{18.2}$ $\mathrm{eV}$ are covered by the TALE fluorescence monocular measurement over 22 months [20], and the energies above $10^{18.2} \mathrm{eV}$ are covered by the TA SD data collected over the 11 year period using the standard TA spectrum analysis and cuts described in the previous section of this work. The lower part of the TA spectrum introduces 3 additional features: the knee structure near $\sim 10^{15.5} \mathrm{eV}$, the low energy ankle at $10^{16.22 \pm 0.02} \mathrm{eV}$, and the second knee at $10^{17.04 \pm 0.04} \mathrm{eV}$. Full details of the TALE measurement and the broken power law fit results to the TALE spectrum can be found in [20]. The overall TA energy scale systematic uncertainty has been previously estimated to be $\sim 20 \%$ [21].

\section{Summary}

In this work, we have updated the TA spectrum result with 11 years of TA SD data. The combined TA SD and TALE spectrum shows 5 features and covers $\sim 5$ orders of magnitude in energy using one energy scale. At the highest energies, we see an evidence for the declination dependence of the TA SD spectrum, the local significance of which appears to increase from 4.0 to $4.7 \sigma$ as we add 4 more years of the TA SD data. The global significance of the effect, with present TA SD data, has been estimated to be $4.3 \sigma$, and we have found that the effect cannot be explained by the systematic uncertainties. The TAx4 surface array expansion [22], which is currently under construction in Millard County, UT, USA, is expected to provide a data sample that should be 

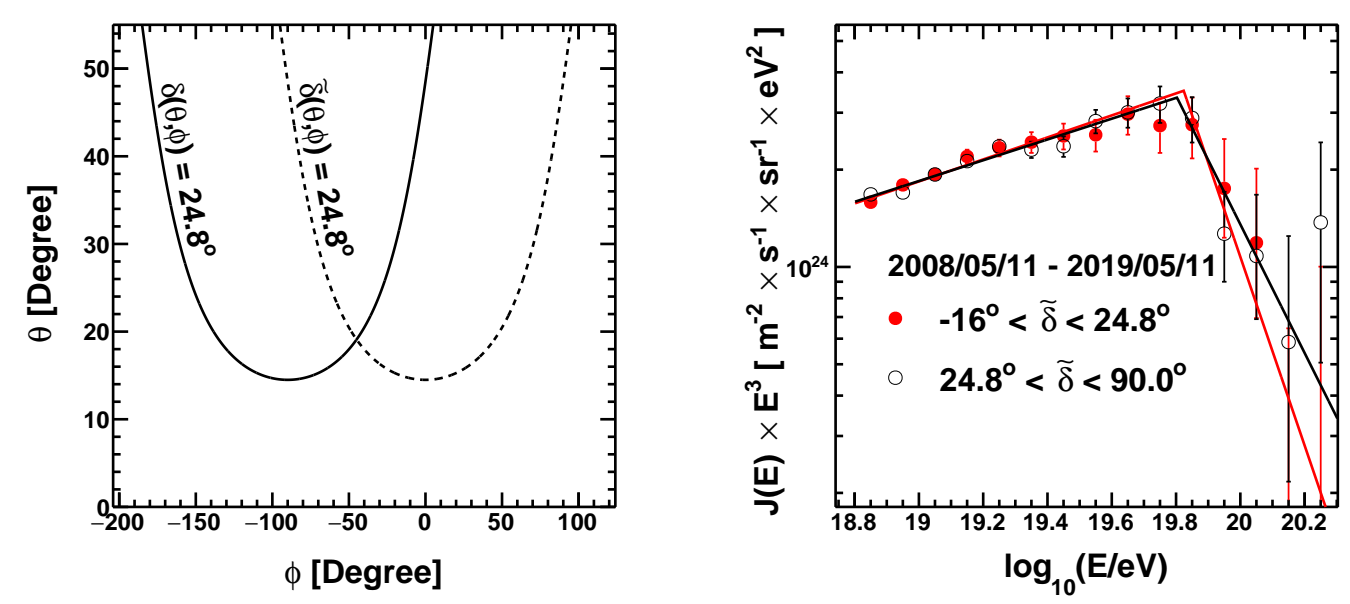

Figure 5: Left: Solid line shows a constant declination contour $\delta(\theta, \phi)=24.8^{\circ}$ in local horizontal coordinates of the TA experiment. Zenith and azimuthal angles of the event arrival direction are labeled by $\theta$ and $\phi$, respectively. The event azimuthal angle $\phi$ is measured in the counterclockwise direction from the direction of East at the TA site. Events in the lower declination band $\left(\delta<24.8^{\circ}\right)$ occur above the solid line while events in the upper declination band $\left(\delta>24.8^{\circ}\right)$ occur below the solid line. One can transform the declination function to represent the equatorial east and west, instead of equatorial north and south by shifting the azimuthal angle by $90^{\circ}: \tilde{\delta}(\theta, \phi)=\delta\left(\theta, \phi-90^{\circ}\right)$. Dotted line represents the contour of constant modified declination, $\tilde{\delta}(\theta, \phi)=24.8^{\circ}$. Points above the dotted line correspond to the equatorial east band, while points below the dotted line correspond to the equatorial west band. Right: TA SD energy spectrum collected over 11 years for the equatorial east part of the data, $\tilde{\delta}<24.8^{\circ}$, and the equatorial west part, $\tilde{\delta}>24.8^{\circ}$. Superimposed are the fits to the broken power law functions. For the equatorial east part, the spectral indices are $-2.66 \pm 0.03$ and $-5.9 \pm 0.9$ before and after the break, respectively, while the break point is at $10^{19.82 \pm 0.03} \mathrm{eV}$. For the equatorial west part, the spectral indices before and after the break are $-2.68 \pm 0.03$ and $-5.0 \pm 0.5$, respectively, and the break point is at $10^{19.80 \pm 0.04} \mathrm{eV}$.

sufficiently large to resolve this effect, and perhaps other anisotropies in the Northern hemisphere, with a $5 \sigma$ global significance.

\section{References}

[1] T. Abu-Zayyad et al., Nucl. Instrum. Meth. A 689 (2012) 87

[2] T. Abu-Zayyad et al., Nucl. Instrum. Meth. A 609 (2009) 227

[3] T. Abu-Zayyad et al., Astropart. Phys. 39-40 (2012) 109

[4] R. U. Abbasi et al., Phys. Rev. Lett. A 100 (2008) 101101

[5] K. Greisen, Phys. Rev. Lett. 16 (1966) 183

[6] G. T. Zatsepin and V. A. Kuz'min, Sov. Phys. JETP Lett. 4 (1966) 114.

[7] V. Berezinsky et al., Phys. Rev. D 74 (2006) 043005.

[8] T. Abu-Zayyad et al., Astrophys. J. 768 (2013) L1

[9] D. Ivanov, "Energy Spectrum Measured by the Telescope Array Surface Detector", doctoral thesis, Rutgers University, New Brunswick, New Jersey, October, 2012. 


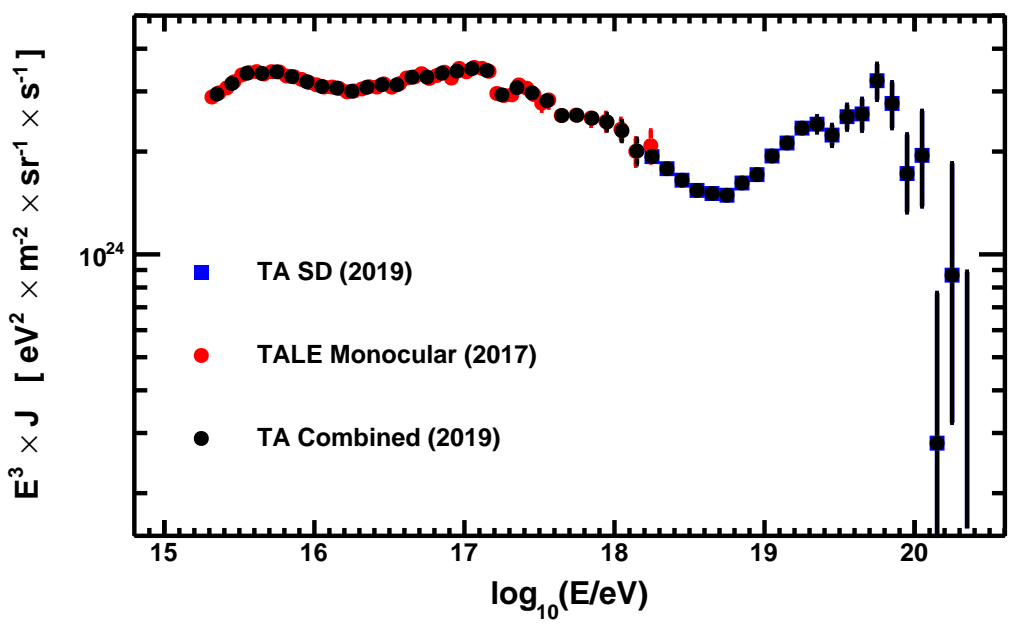

Figure 6: Combined TA Spectrum (black circles) derived using 22 months of TALE monocular data (red circles) and 11 years of the TA SD data analyzed using the standard TA SD spectrum analysis and cuts (blue squares). The broad knee feature occurs near $10^{18.5} \mathrm{eV}$, the low energy ankle is measured at $10^{16.22 \pm 0.02} \mathrm{eV}$, the second knee occurs at $10^{17.04 \pm 0.04} \mathrm{eV}$, the ankle is measured at $10^{18.69 \pm 0.01} \mathrm{eV}$, and the cutoff is measured at $10^{19.81 \pm 0.03} \mathrm{eV}$.

[10] K. Shinozaki et al., Nucl. Phys. Proc. Suppl. 136 (2004) 18

[11] J. Knapp and D. Heck, Nachr. Forsch. zentr. Karlsruhe 30 (1998) 27.

[12] S. Ostapchenko, Nucl. Phys. Proc. Suppl. 151 (2006) 143

[13] M. Kobal, Astropart. Phys. 259-273, 15 (2001)

[14] B. T. Stokes, R. Cady, D. Ivanov, J. N. Matthews and G. B. Thomson, Astropart. Phys. 35 (2012) 759

[15] V. Verzi, for the Telescope Array and Pierre Auger Collaborations, in Proc. UHECR 2016, Kyoto, Japan (2016).

[16] D. Ivanov, for the Telescope Array Collaboration, in Proc. UHECR 2018, Paris, France (2018).

[17] J. Hersil, I. Escobar, D. Scott, G. Clark and S. Olbert, Phys. Rev. Lett. 6, 22 (1961)

[18] D. Ivanov, for the Telescope Array Collaboration, in Proc. ICRC 2017, Busan, Korea (2017).

[19] D. Ivanov, for the Telescope Array and Pierre Auger Collaborations, in Proc. UHECR 2018, Paris, France (2018).

[20] R. U. Abbasi et al. [Telescope Array Collaboration] Astrophys. J. 865, 74 (2018)

[21] Y. Tsunesada, T. AbuZayyad, D. Ivanov, G. Thomson, T. Fujii and D. Ikeda, for the Telescope Array Collaboration, PoS ICRC 2017, 535 (2018).

[22] E. Kido, for the Telescope Array Collaboration, "The TAx4 experiment" Contribution ID CRI199, ICRC-2017, Bexco, Busan, Korea 